\title{
Bulging fontanelle in a term newborn: A case report
}

\author{
Eirini Koutsounaki*, Xenia Bitouni, Kaliopi Drakaki, Anastasia Kapetanaki, George Liosis and Iraklis Salvanos \\ Helena Venizelou General Hospital of Athens, NICU, Greece
}

\begin{abstract}
We describe a case of a female term neonate born after a normal pregnancy and a caesarian section due to breach orientation. Because of meconium stained and compromised clinical condition the newborn was admitted to the NICU, immediately, after birth, for observation. Apart from a bulging anterior fontanelle and mildly hypotonic muscular tone, no other clinical signs were referred. The serial cranial ultrasound showed moderate dilation of the lateral and third ventricles imposing the need for further investigation. The magnetic resonance imaging showed major cerebellar hemorrhage, as well as posterior fossa subdural and subarachnoid hemorrhages. Noninvasive MRI vascular imaging (MRA, MRV) did not show any evidence of vascular dysplasia or thrombosis. The laboratory investigation of thrombophilia noted heterozygous prothrombin 20210A mutation.
\end{abstract}

\section{Case presentation}

A female infant was born at 41 weeks of gestational age by cesarean section due to breach presentation to a 40 year old, gravida I, para I mother. The mother, otherwise healthy, had gestational diabetes limited by dietary control. She had a negative history for thrombotic events and received appropriate prenatal care and had normal routine prenatal ultrasound scanning during pregnancy (first, second and third trimester). During the $\mathrm{c}$-section, meconium-stained amniotic fluid was noted. The neonate's birth weight was $2.9 \mathrm{~kg}$, head circumference 34 $\mathrm{cm}$, and length $47 \mathrm{~cm}$. Right after birth, the infant presented with poor muscle tone, inadequate respiratory effort and bradycardia, responded well to positive pressure applied by T-piece and recovered within 30 seconds. Apgar scores was 4 and 8 at $1^{\text {st }}$ and $5^{\text {th }}$ minute respectively. Cord gases were unremarkable: $\mathrm{pH} 7.29 /$ base excess $-5.2 \mathrm{mmol} / \mathrm{L}$, $\mathrm{HCO} 317.8 \mathrm{mmol} / \mathrm{L}$ and lactate $24 \mathrm{mg} / \mathrm{dl}$. The infant was transferred to the NICU for observation.

Physical examination revealed mild hypotonia, head lag, intact neonatal reflexes and no seizures were detected. Bulging anterior fontanelle was profound, a sign presented persistently.

The infants' cardiorespiratory status was stable. No oxygen therapy was provided. Oral feedings was initiated soon after the first hours of observation.

A cranial ultrasound was performed due to the persistently bulging fontanelle on the second day of life and repeated the following day illustrating moderate dilatation of the lateral and third ventricles (Figure 1). Unfortunately, the mastoid fontanelle approach although searched for, was typically closed in this term neonate. Laboratory tests were performed for infectious diseases and inborn error of metabolism, which were unremarkable and also a Magnetic Resonance Imaging (MRI) was scheduled and performed on the day 7 of life. The T1 and the T2 weighted MRI imaging was inconclusive (Figure 2), but diffusion-weighted magnetic resonance imaging (DWI) revealed a major cerebellar hemorrhage, as well as posterior fossa subdural hemorrhage and subarachnoid hemorrhage (Figure 3). In order to exclude any vascular malformations, such as superior cerebellar artery aneurism or dysplasia and/or thrombosis, further investigation was conducted. Magnetic Resonance Angiography (MRA) and Magnetic
Resonance Venography (MRV) on the 20th day of life illustrated normal appearance of the intracranial vessels (Figure 4). Furthermore, laboratory investigation of thrombophilia was performed and noted heterozygous prothrombin 20210 A mutation.

\section{Discussion}

Although, cerebellar hemorrhage in prematures is a quite often condition, it is a sparsely recognized condition in the term neonates. These lesions can be related to significant neurodevelopmental consequences. The main risk factors for infant brain injury including asphyxia, hemorrhagic predisposition and malformations are shown in the table 1. Clinical findings in such cases include apnea, bradycardia and a decline in hematocrit, which interestingly were lucking from our patient.

In the present case, the leading clinical sign that led to the diagnosis was the infant's bulging anterior fontanelle. Several risk factors were investigated, both in the mother and the neonate, which might

Table 1. Peripartum cerebellar hemorrhage in the term neonate

\begin{tabular}{|l|l|}
\hline Perinatal asphyxia & Congenital hydrocephalus \\
\hline Urgent c- section & Cerebellar malformations \\
\hline Trauma during labor and delivery & Nervous system vascular malformations \\
\hline Respiratory distress syndrome & $\begin{array}{l}\text { Posterior Inferior Cerebellar Artery PICA } \\
\text { aneurysm }\end{array}$ \\
\hline Pneumothorax & Congenital medulloblastoma \\
\hline Patent ductus arteriosus & Fetal anemia \\
\hline Congenital Diaphragmatic Hernia & Bleeding secondary to thrombus formation \\
\hline Sepsis & Bleeding disorders \\
\hline Maternal chorioamnionitis, preeclampsia & Congenital thrombocytopenia \\
\hline Intrauterine infections (CMV) & \\
\hline
\end{tabular}

Correspondence to: Eirini Koutsounaki, Neonatologist, Helena Venizelou General Hospital of Athens, NICU, Greece, E-mail: eirkou@hotmail.com

Key words: peripartum cerebellar hemorrhage, bulging fontanelle, newborn, term

Received: October 16, 2017; Accepted: October 27, 2017; Published: October 30,2017 


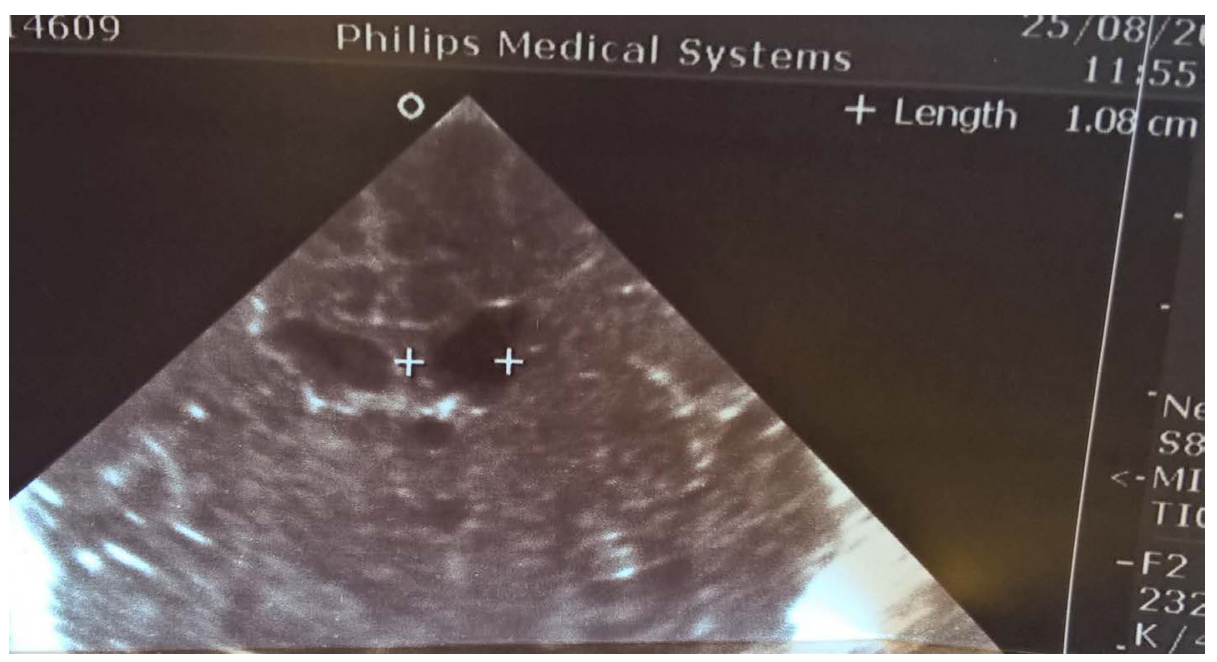

Figure 1. Cranial ultrasound focusing on moderate ventricles' dilatation

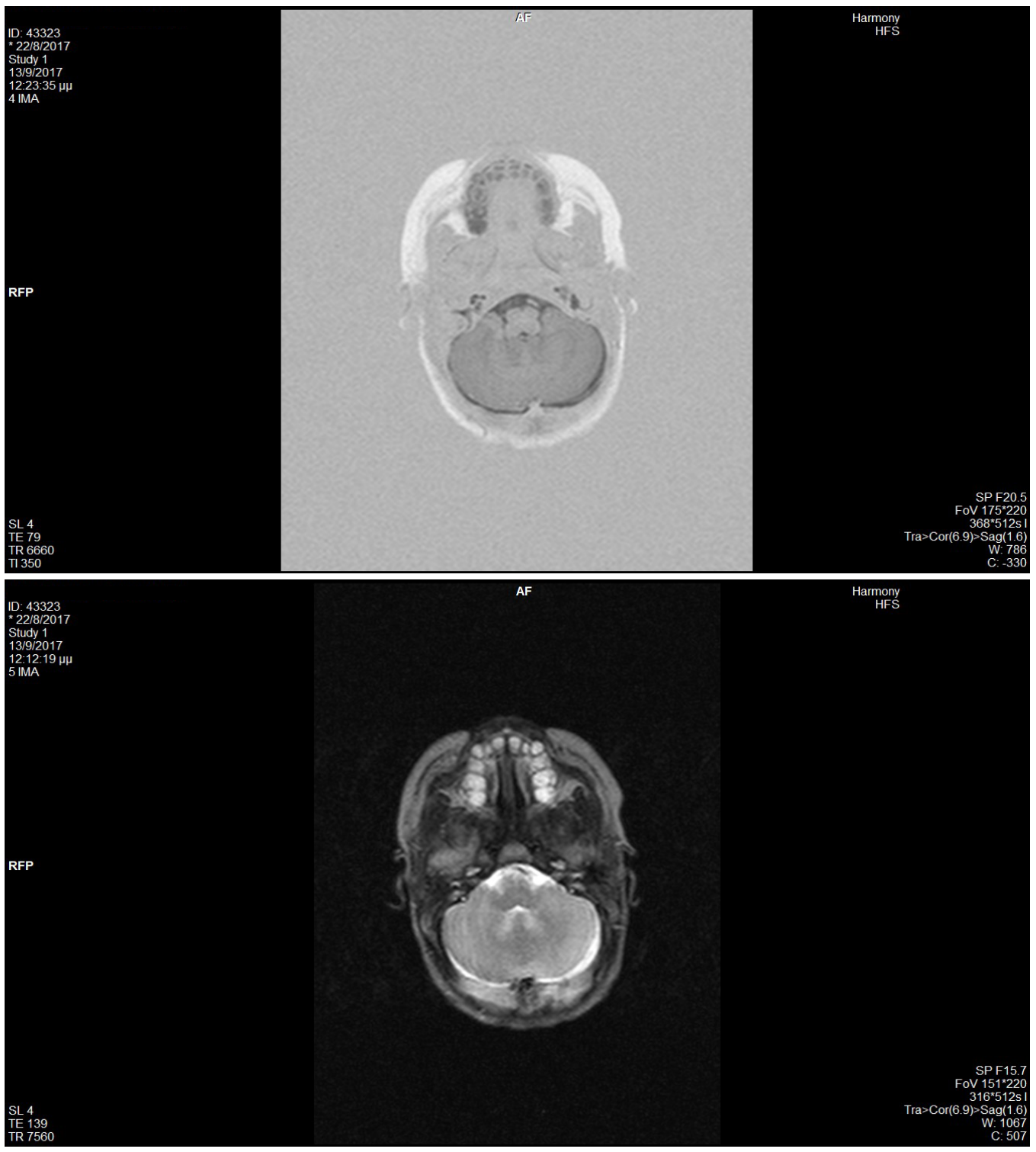

Figure 2. i), ii). The $\mathrm{T} 1$ and the $\mathrm{T} 2$ weighted MRI imaging was inconclusive 


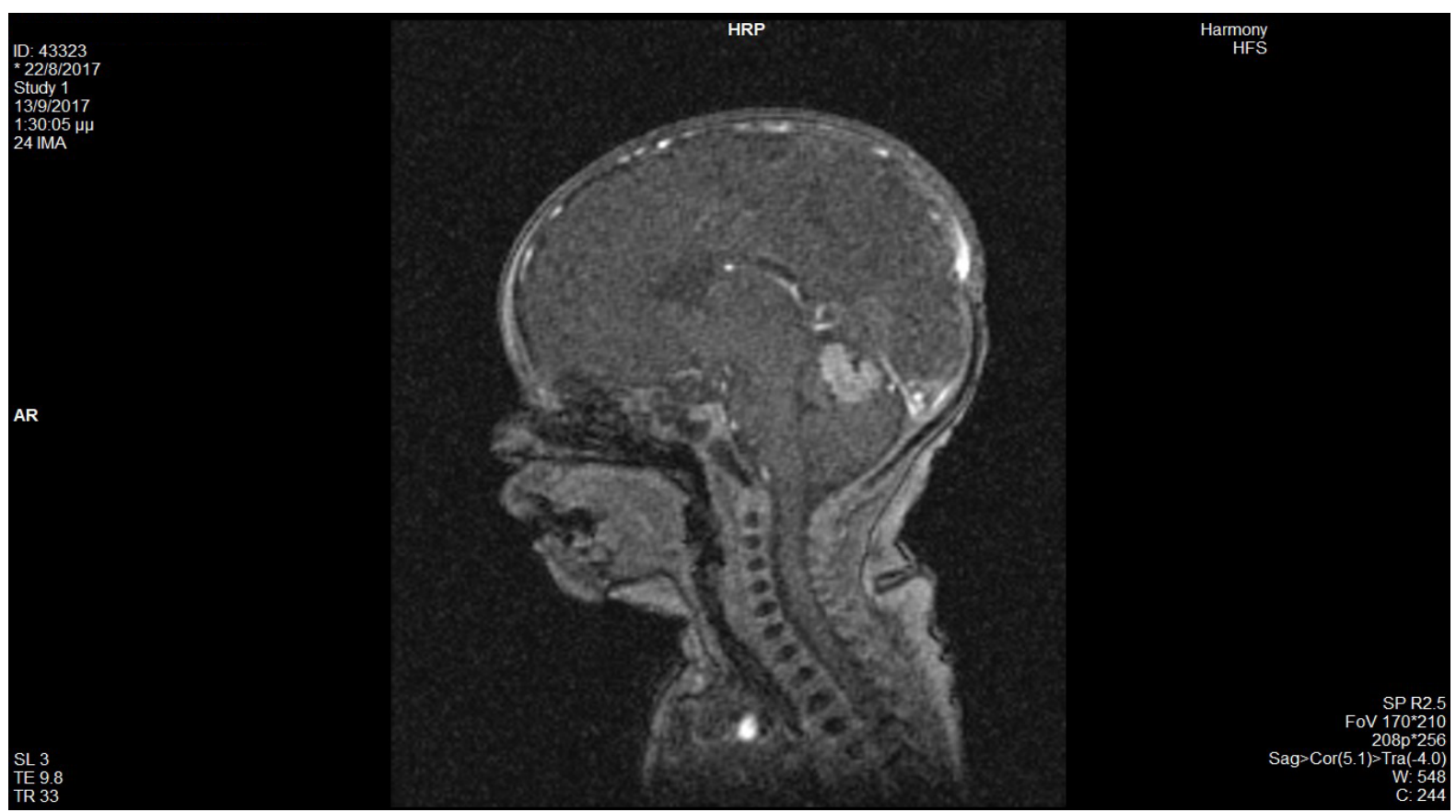

Figure 3. Diffusion-Weighted magnetic resonance imaging (DWI) revealed a major cerebellar hemorrhage.

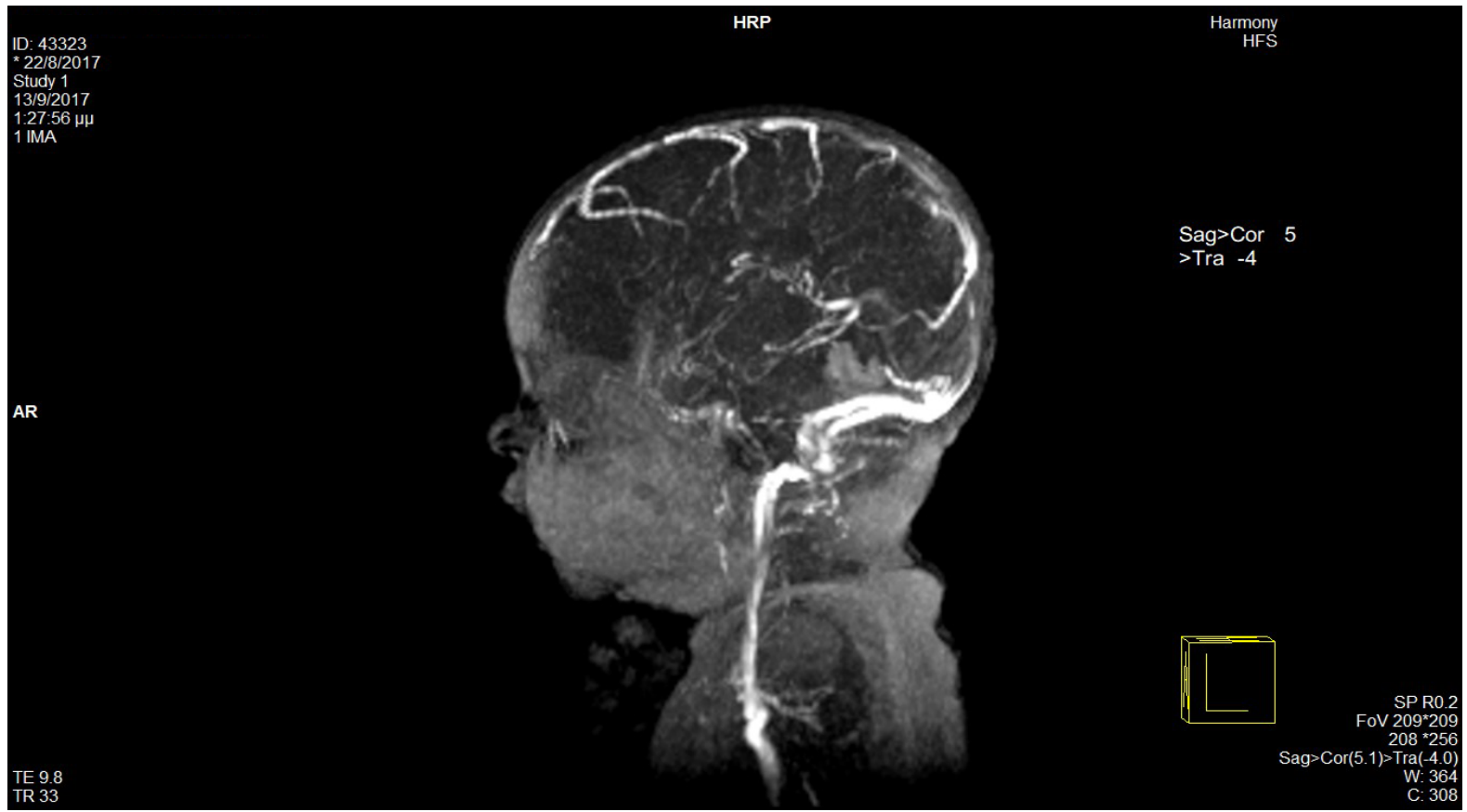

Figure 4. Magnetic Resonance Angiography (MRA) and Magnetic Resonance Venography (MRV), illustrated normal appearance of the intracranial vessels

have contributed to this sparsely recognized condition in the term neonates. We estimate that perinatal stress and thrombophilia genetic predisposition have played a role. In this case, thorough investigation revealed heterozygous prothrombin 20210A mutation that may have been the main predisposing factor.

\section{Conclusion}

Although cerebellar hemorrhage $(\mathrm{CH})$ is a well-known common complication of prematurity, this case highlights that cerebellar hemorrhage might also be more common in the term infant than previous thought. It is also remarkable to emphasize on the classical MRI images, such as T1 and T2 weighted, might not be sufficient to reveal cerebellar bleeding and additional studies of diffusion as well as MRA and MRV were needed in order to provide the diagnosis. Infants with $\mathrm{CH}$ are at high risk for neurodevelopmental disability. Thus, clinicians should be alert for this disorder and its predisposing factors

\section{References}

1. Bergman I, Bauer RE, Barmada MA, Latchaw RE, Taylor HG, et al. (1985) Intracerebral Hemorrhage in the Full-Term Neonatal Infant. Pediatrics 75: 488-96. [Crossref] 
2. Poretti A, Boltshauser E, Huisman TA (2016) Prenatal Cerebellar Disruptions: Neuroimaging Spectrum of Findings in Correlation with Likely Mechanisms and Etiologies of Injury. Neuroimaging Clin NAm 26: 359-72. [Crossref]
3. Tanriverdi SR, Turhan T, Uygur O, Koroglu OA, Yalaz M, et al. (2013) Endoscopic evacuation of cerebellar hematoma in a term newborn. Brain Dev 35: 881-4. [Crossref]

Copyright: $@ 2017$ Koutsounaki E. This is an open-access article distributed under the terms of the Creative Commons Attribution License, which permits unrestricted use, distribution, and reproduction in any medium, provided the original author and source are credited. 